\title{
Article
}

\section{Metal Halide Perovskite Nanosheet for X-Ray High-Resolution Scintillation-Imaging Screens}

Yuhai Zhang, Ruijia Sun, Xiangyu Ou, Kaifang Fu, Qiushui Chen, Yuchong

Ding, Liang-Jin Xu, Lingmei Liu, Yu Han, Anton V. Malko, Xiaogang Liu,

Huanghao Yang, Osman M. Bakr, Hong Liu, and Omar F. Mohammed

ACS Nano, Just Accepted Manuscript • DOI: 10.1021/acsnano.8b09484 • Publication Date (Web): 05 Feb 2019

Downloaded from http://pubs.acs.org on February 10, 2019

\section{Just Accepted}

"Just Accepted" manuscripts have been peer-reviewed and accepted for publication. They are posted online prior to technical editing, formatting for publication and author proofing. The American Chemical Society provides "Just Accepted" as a service to the research community to expedite the dissemination of scientific material as soon as possible after acceptance. "Just Accepted" manuscripts appear in full in PDF format accompanied by an HTML abstract. "Just Accepted" manuscripts have been fully peer reviewed, but should not be considered the official version of record. They are citable by the Digital Object Identifier (DOI®). "Just Accepted" is an optional service offered to authors. Therefore, the "Just Accepted" Web site may not include all articles that will be published in the journal. After a manuscript is technically edited and formatted, it will be removed from the "Just Accepted" Web site and published as an ASAP article. Note that technical editing may introduce minor changes to the manuscript text and/or graphics which could affect content, and all legal disclaimers and ethical guidelines that apply to the journal pertain. ACS cannot be held responsible for errors or consequences arising from the use of information contained in these "Just Accepted" manuscripts. 


\title{
Metal Halide Perovskite Nanosheet for X-Ray High-Resolution Scintillation-Imaging Screens
}

\author{
Yuhai Zhang, ${ }^{1,2}{ }^{*}$ Ruijia Sun, ${ }^{1}$ Xiangyu Ou, ${ }^{3}$ Kaifang Fu, ${ }^{1}$ Qiushui Chen, ${ }^{4}$ \\ Yuchong Ding, ${ }^{5}$ Liang-Jin $\mathrm{Xu},{ }^{2}$ Lingmei Liu, ${ }^{6}$ Yu Han, ${ }^{6}$ Anton V. Malko, \\ Xiaogang Liu, ${ }^{4}$ Huanghao Yang, ${ }^{3}$ Osman M. Bakr, ${ }^{2}$ Hong Liu, ${ }^{1}{ }^{*}$ and Omar F. \\ Mohammed, ${ }^{2 *}$
}

${ }^{1}$ Institute for Advanced Interdisciplinary Research (iAIR), University of Jinan, Jinan 250022, Shandong, China

${ }^{2}$ King Abdullah University of Science and Technology, Division of Physical Sciences and Engineering, Thuwal 23955-6900, Saudi Arabia

${ }^{3}$ MOE Key Laboratory for Analytical Science of Food Safety and Biology, State Key Laboratory of Photocatalysis on Energy and Environment, College of Chemistry, Fuzhou University, Fuzhou 350002 China.

${ }^{4}$ Department of Chemistry, National University of Singapore, Singapore 117543, Singapore

${ }^{5}$ Research \& Development Center of Material and Equipment, China Electronics Technology Group, Corporation No. 26 Research Institute, Chongqing 400060, China ${ }^{6}$ King Abdullah University of Science and Technology (KAUST), Advanced Membranes and Porous Materials Center, Physical Sciences and Engineering Division, Thuwal 23955-6900, Saudi Arabia.

${ }^{7}$ Department of Physics, The University of Texas at Dallas, Richardson, TX, 75080, USA

\section{Corresponding Authors}

ifc_zhangyh@ujn.edu.cn, ifc_liuh@ujn.edu.cn, omar.abdelsaboor@kaust.edu.sa 


\section{ABSTRACT}

Scintillators, which are capable of converting ionizing radiation into visible photons, are an integral part of medical, security, and commercial diagnostics technologies such as X-ray imaging, nuclear cameras, and computed tomography. Conventional scintillator fabrication typically involves high-temperature sintering, generating agglomerated powders or large bulk crystals, which pose major challenge for device integration and processability. On the other hand, colloidal quantum dot scintillators cannot be cast into compact solid films with the necessary thickness required for most X-ray applications. Here, we report the room-temperature synthesis of a colloidal scintillator comprising $\mathrm{CsPbBr}_{3}$ nanosheets of large concentration (up to $150 \mathrm{mg} / \mathrm{mL}$ ). The $\mathrm{CsPbBr}_{3}$ colloid exhibits a higher light yield $(\sim 21000$ photons $/ \mathrm{MeV})$ than the commercially available Ce:LuAG single-crystal scintillator ( 18000 photons/MeV). Scintillators based on these nanosheets display both strong radioluminescence (RL) and long-term stability under X-ray illumination. Importantly, the colloidal scintillator can be readily cast into a uniform crack-free large-area film $\left(8.5 \times 8.5 \mathrm{~cm}^{2}\right.$ in area $)$ with the requisite thickness for high-resolution X-ray imaging applications. We showcase prototype applications of these high-quality scintillating films as X-ray imaging screens for a cellphone panel and a standard central processing unit (CPU) chip. Our radiography prototype combines large-area processability with high resolution and a strong penetration ability to sheath materials, such as resin and silicon. We reveal an energy transfer process inside those stacked nanosheet solids that is responsible for their superb scintillation performance. Our findings demonstrate a large-area solution- 
processed scintillator of stable and efficient RL as a promising approach for low-cost radiography and X-ray imaging applications.

\section{TOC Graphic}

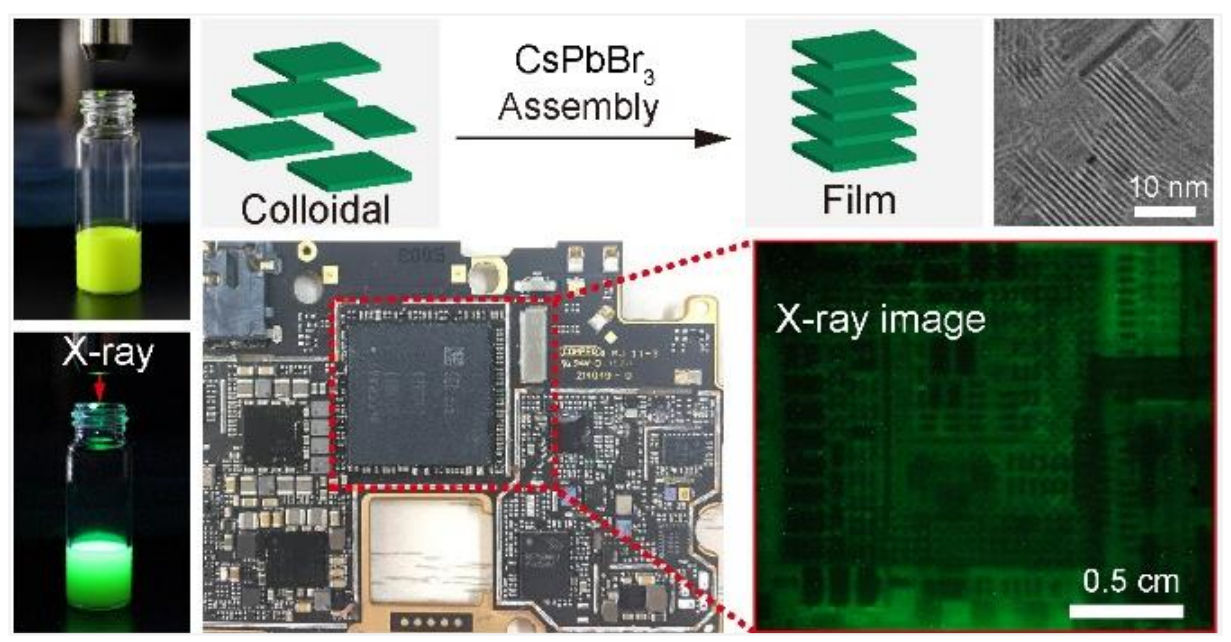

Keywords: perovskite, colloidal scintillator, self-assembly, X-ray imaging, energy transfer, nanosheets 
Scintillating materials are widely used for radiation-detection applications in many fields, including interstellar particle detection, X-ray security, nuclear cameras, and computed tomography, due to their ability to convert ionizing radiation into visible or ultraviolet photons. ${ }^{1,2}$ Conventional scintillators, such as CsI(Tl) crystals and ceriumactivated glass, are usually synthesized under high-temperature conditions, ${ }^{3,4}$ which is neither cost-effective nor user-friendly. Additionally, the resulting powder scintillator suffers from severe agglomeration while a precisely sliced single crystal is required to be easily incorporated into device architectures. ${ }^{5,6}$

An ideal scintillator with a controllable thickness should be solution-processable. Organic dye solutions, such as $p$-terphenyl $\left(\mathrm{C}_{18} \mathrm{H}_{14}\right)$, PBD $\left(\mathrm{C}_{20} \mathrm{H}_{14} \mathrm{~N}_{2} \mathrm{O}\right)$, and PPO $\left(\mathrm{C}_{15} \mathrm{H}_{11} \mathrm{NO}\right)$, have been used as scintillators to detect neutrons. ${ }^{7-9}$ However, the detection efficiency is substantially low due to their low stopping power because of their low atomic number constituents. Moreover, organic dyes are intrinsically susceptible to both the photobleaching effect and oxygen quenching, ${ }^{10}$ hindering their practical application in many areas. It is worth pointing out that hybrid perovskite films incorporating heavy-metal ions became an excellent candidate for X-ray detection. ${ }^{11-17}$ For instance, all-inorganic perovskite nanocrystals have recently emerged as promising semiconductor materials for efficient scintillators due to their high stopping power, low detection limit, and multicolor RL. ${ }^{18}$ However, these nanocrystals are difficult to cast into compact solid form films with the requisite thickness (due to the long penetration depth of X-rays) and area sizes for commercially viable applications. Thus, only small device areas were possible to be fabricated and tested. $1,11,14,15,19$ 
Here, we report the room-temperature synthesis of a high-quality scintillator in both colloidal and solid film forms, designed and self-assembled from highly concentrated solutions of perovskite nanosheets. The colloidal form of concentrated $\mathrm{CsPbBr}_{3}$ nanosheets exhibits equivalent brightness of RL to the film counterpart and shows longterm stability under both storage and X-ray exposure conditions. Due to their high concentration and nanosheet morphology, our scintillators are readily solutionprocessable and tend to assemble into a crack-free film over a large area up to $72 \mathrm{~cm}^{2}$, allowing for high-resolution $(<0.21 \mathrm{~mm}) \mathrm{X}$-ray imaging applications. Finally, we used time-resolved photoluminescence spectroscopy to reveal an energy transfer process inside those stacked nanosheets that enhances the scintillation performance beyond what is typically expected in nanocrystal scintillators.

\section{RESULTS AND DISCUSSION}

The $\mathrm{CsPbBr}_{3}$ nanosheets were synthesized using a modified coprecipitation method. ${ }^{20}$ Briefly, an excessive amount of $\mathrm{PbBr}_{2}$ precursor was injected into a cesium acetate precursor at room temperature under an ambient atmosphere (see the Supporting Information for further details). A bright green color appeared immediately, indicating the formation of $\mathrm{CsPbrr}_{3}$ nanosheets. Conventionally, the purification process of $\mathrm{CsPbBr}_{3}$ nanocrystals requires breaking the colloidal stability by the addition of a poor solvent, ethyl acetate for example, which inevitably induces severe degradation of the particles. $^{21,22}$ In this work, however, the nanosheets could be easily collected by lowspeed centrifugation $(<4000 \mathrm{rpm})$ without using any polar solvents. The collected 
nanosheets were dispersed in toluene with a high concentration of up to $150 \mathrm{mg} / \mathrm{mL}$, and the resulting colloid could be kept in a capped vial for one month without any noticeable degradation (Figure S1).

a)

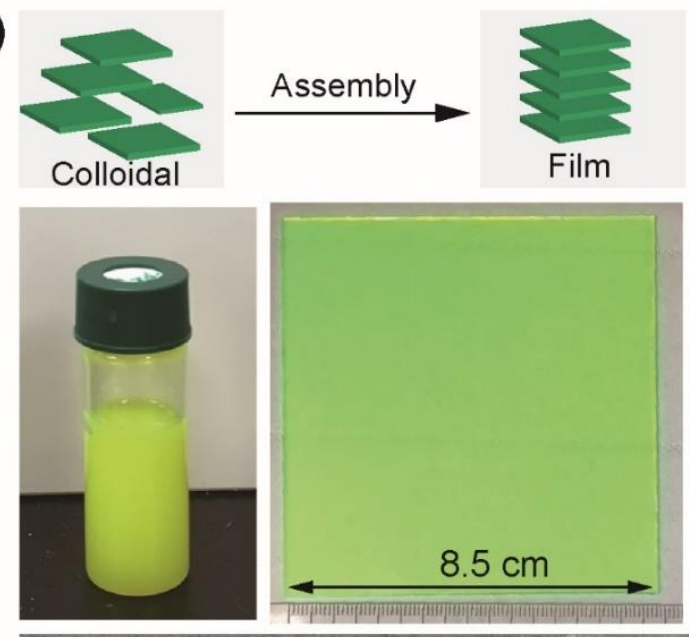

e)

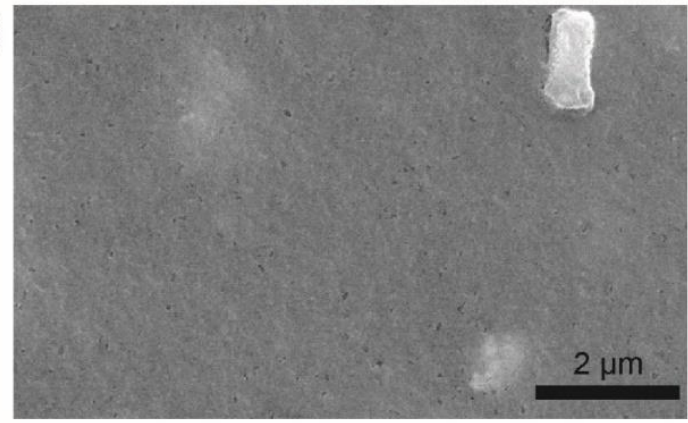

b)
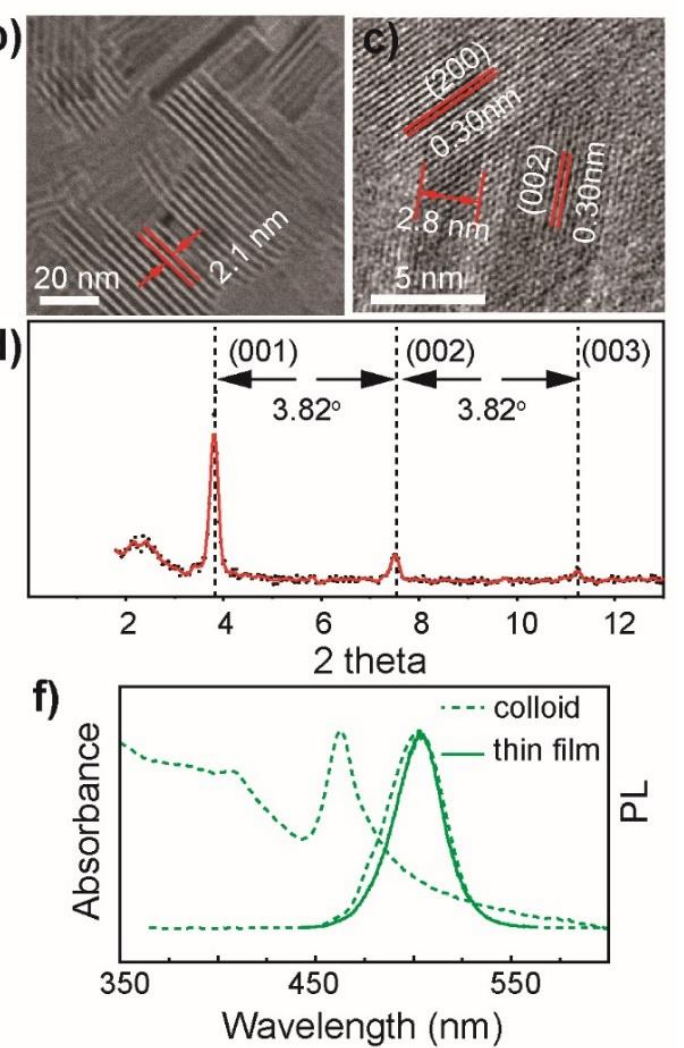

Figure 1. a) Schematic showing the self-assembly process of $\mathrm{CsPbBr}_{3}$ nanosheets. The bottom panel shows the photographs of a heavily loaded colloid $(150 \mathrm{mg} / \mathrm{mL})$ in toluene and a wafer-sized thin film on a glass slide. b) TEM image of the assembled $\mathrm{CsPbBr}_{3}$ nanoplatelets, featuring an interplane spacing of $2.1 \mathrm{~nm}$. c) HRTEM image of the assembly, showing a clear lattice fringe of (200) facets. d) Low-angle XRD diffraction pattern of the assembled $\mathrm{CsPbBr}_{3}$ thin film, showing a series of interplane diffraction peaks of the stacked $\mathrm{CsPbBr}_{3}$ nanoplatelets. The peak spacing of $3.82^{\circ}$ suggests an interplane spacing of $2.32 \mathrm{~nm}$, in line with the TEM results. e) SEM image showing the flatness of the thin film. f) Steady-state absorption and photoluminescence (excitation at $365 \mathrm{~nm}$ ) spectra of the colloid and thin-film samples. 
The large-area thin film was fabricated through a self-assembly process of $\mathrm{CsPbBr}_{3}$ nanosheets. Typically, the concentrated colloid was drop cast on a clean glass substrate under ambient conditions and allowed to dry naturally. A crack-free perovskite thin film was obtained in 12 minutes, showing a green tint under ambient light (Figure 1a). The TEM image reveals the face-to-face packing manner of $\mathrm{CsPbBr}_{3}$ nanosheets with an interplane gap of $2.1 \mathrm{~nm}$ (Figure 1b). Moreover, the HRTEM image shows the lattice fringes of both a lateral nanosheet and an aligned nanosheet, featuring a (002) d-spacing of $0.30 \mathrm{~nm}$ (Figure 1c). Importantly, we were able to accurately measure both the thickness of the nanosheets $(2.8 \sim 4.2 \mathrm{~nm})$ and the gap between them $(2.1 \mathrm{~nm})$. Considering the lattice constant $(0.59 \mathrm{~nm})$ of a single unit cell, we were able to identify the number of monolayers (5-7 layers) comprising an individual nanosheet, which was in good agreement with both the HRTEM observation and the Scherrer broadening effect (Figure S2). The thin film was rather flat, featuring a surface roughness of approximately $13 \mathrm{~nm}$ and a thickness of approximately $4.0 \mu \mathrm{m}$ (Figure 1e, Figure S3), which is critical for high-resolution radiography.

To further confirm the self-assembly nature of the thin film, low-angle powder XRD was conducted. The XRD pattern shows three peaks with an equal spacing of $3.82^{\circ}$, indicating that the slit of the superstructure is approximately $2.3 \mathrm{~nm}$. This value is in line with our result obtained from HRTEM $(2.1 \mathrm{~nm})$, suggesting that there are two layers of passivating ligands (octylamine or octanoic acid) occluding the gap. It is worth noting that the result is in good agreement with the value obtained from the reported relationship $\mathrm{d}(\AA)=8.06+1.59 \times \mathrm{n}$, where $\mathrm{n}$ is the number of carbon atoms in the 
alkylamine (for octylamine or octanoic acid, $n=8$ ). ${ }^{23}$ The thin film showed intense luminescence centered at $515 \mathrm{~nm}$ under $365-\mathrm{nm}$ excitation, which is slightly spectrally redshifted compared to that of the colloidal counterpart. Interestingly, the absorption of the colloid exhibited an excitonic peak at $460 \mathrm{~nm}$, together with a long tail extending to $570 \mathrm{~nm}$ (Figure 1f). This broadening can be ascribed to the inhomogeneous distribution of the nanosheet thickness, as evidenced by HRTEM.
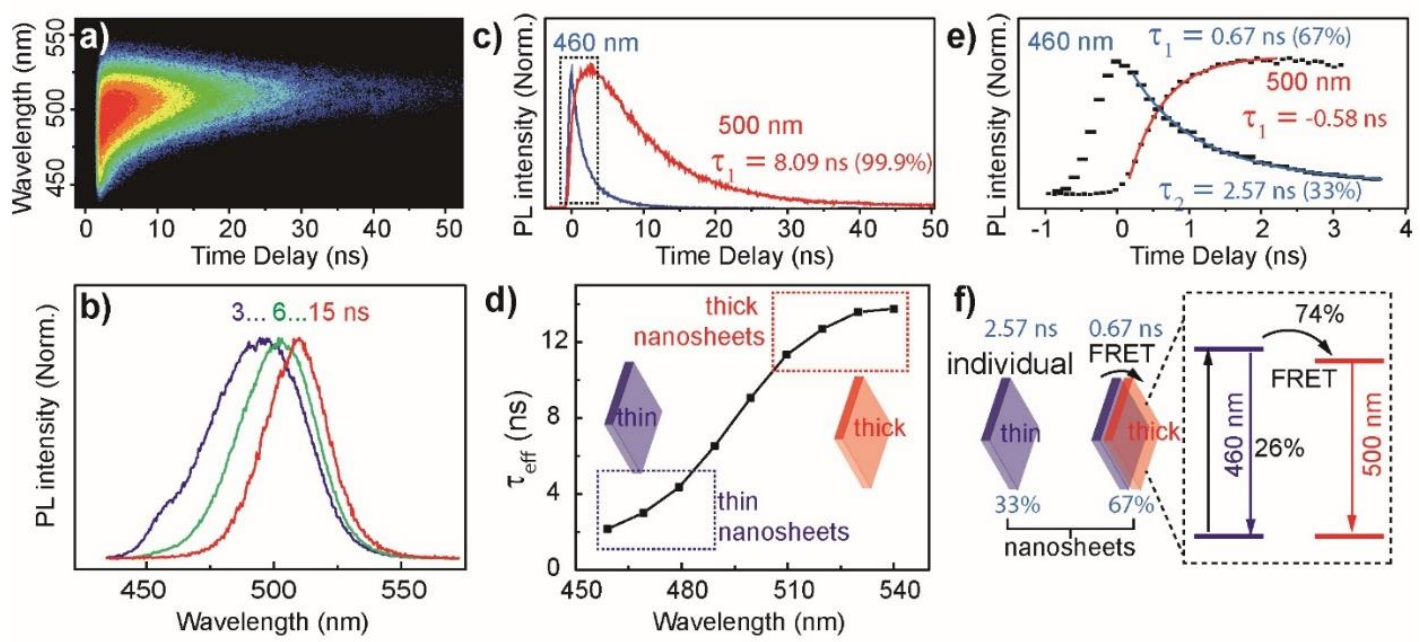

Figure 2. Streak camera measurement of transient $\mathrm{PL}$ from the thin film of the $\mathrm{CsPbBr} 3$ nanosheet assembly ( $\mathrm{Pb} / \mathrm{Cs}: 5 / 1)$. a) 2D pseudocolor transient emission map, excitation at $410 \mathrm{~nm}$, flux at $1 \mathrm{pJ} / \mathrm{cm}^{2}$. b) Transient PL profiles at $3 \mathrm{~ns}, 6 \mathrm{~ns}$, and $15 \mathrm{~ns}$, showing the recombination center shifting of excitons. PL decay traces extracted at 460 and 500$\mathrm{nm}$ windows $( \pm 5 \mathrm{~nm})$. c) Magnified image of PL decay traces in the first $3 \mathrm{~ns}$, where the building-up lifetime of 500-nm emission is comparable to the short component of 460-nm emission, indicating an efficient energy transfer. The dashed box highlighted the building-up stage of 500-nm emission. d) The effective lifetime was plotted against the wavelength, with two plateau lifetimes (dashed box) ascribable to thin and thick nanosheets, respectively. e) Magnified image of PL decay traces in the first $3 \mathrm{~ns}$, where the building-up lifetime of 500-nm emission is comparable to the short component of 460-nm emission, indicating an efficient energy transfer. f) Schematic showing the energy transfer process from thin to thick nanosheets, with a FRET efficiency of $74 \%$. 
The coexistence of both thin and thick $\mathrm{CsPBr}_{3}$ nanosheets, as indicated by HRTEM, implies an energy transfer process inside the stacked thin film. To quantitatively evaluate the energy transfer efficiency, we used a streak-camera system to collect the transient PL signal in both wavelength and time domains (Figure 2a). The time-delayed PL profile shows a clear redshift from $460 \mathrm{~nm}$ to $510 \mathrm{~nm}$, indicating that the exciton recombination center shifted from a high energy level to a low one in $15 \mathrm{~ns}$ (Figure 2b). The PL decay trace at $500 \mathrm{~nm}$ shows a nearly monoexponential decay with a lifetime of $8.09 \mathrm{~ns}$ (Figure 2b). In stark contrast, the PL decay trace at $460 \mathrm{~nm}$ comprises both a short component of $0.67 \mathrm{~ns}(67 \%)$ and a long component of $2.57 \mathrm{~ns}(33 \%)$ (Figure 2, e and f). The long component is likely the intrinsic excited-state lifetime of the thin nanosheets (donor), which are isolated from the thick ones (acceptor), with a lifetime value similar to that of five-layer nanoplatelets, as reported by Akkerman et al. ${ }^{24} \mathrm{On}$ the other hand, the short component can be attributed to the energy transfer from the thin to the thick nanosheets.

To validate the assumption that the short-component lifetime of 460-nm emission is due to FRET, we fitted the rising time of 500-nm emission (Figure 2e). Indeed, the rise time of $0.58 \mathrm{~ns}$ at $500 \mathrm{~nm}$ (acceptor) is quite similar to the short component of 0.67 ns at $460 \mathrm{~nm}$ (donor), confirming the abovementioned assumption about FRET. ${ }^{25}$ It is worth noting that the effective lifetime is wavelength dependent with two plateaus, suggesting the existence of both thin and thick nanosheets (Figure 2d). Now, the assembly can be viewed as a donor-acceptor system where the donor has an intrinsic 
lifetime of $2.57 \mathrm{~ns}\left(\tau_{\mathrm{d}}\right)$, and the donor-acceptor assembly has a lifetime of $0.67 \mathrm{~ns}\left(\tau_{\mathrm{da}}\right)$. The FRET efficiency (E) can be calculated by the following equation: ${ }^{26}$

$$
\mathrm{E}=1-\frac{\tau_{d a}}{\tau_{d}}=74 \%
$$

In the thin film of the stacked nanosheet assembly, the donor-acceptor distance (r) is equivalent to the interplane distance, approximately $2.3 \mathrm{~nm}$, as indicated by the smallangle XRD measurement. By using equation (2), we calculated the Förster distance $\left(\mathrm{R}_{0}\right)$ of $\mathrm{CsPbr}_{3}$ nanosheets to be $2.74 \mathrm{~nm}^{27-29}$

$$
\mathrm{E}=\frac{1}{1+\left({ }^{r} / R_{0}\right)^{6}}
$$

Next, we investigated the RL of both colloidal and solid forms of $\mathrm{CsPbr}_{3}$ nanosheets, as well as the transformation process. As shown in Figure 3a and Figure $4 \mathrm{~b}$, the concentrated colloidal nanosheets showed bright green luminescence under Xray excitation as well as the ensuing thin film. In fact, most perovskite nanocrystals tend to compromise on PLQY after colloid-solid transformation due to liganddetachment induced trap states. For example, due to the ligand loss, the $\mathrm{CsPbBr}_{3}$ tethered with oleic-acid ligands dramatically drops its original PLQY from 56 to $18 \%$ after solidification. ${ }^{30,31}$ The stacked nanosheets in this work, however, retained a high PLQY of up to $63 \%$, mainly because the ligands tethered on nanosheets were well shielded among the assembly. 

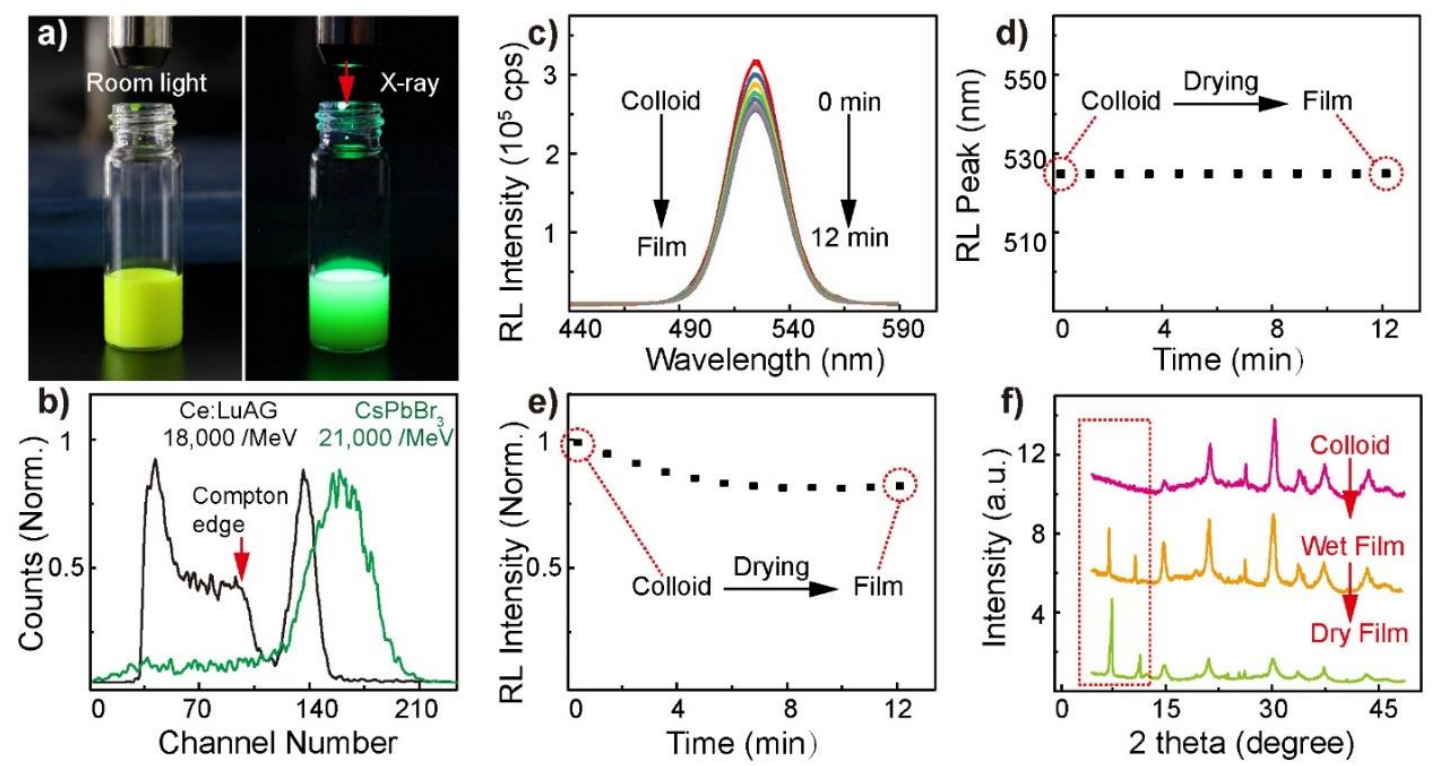

Figure 3. $\mathrm{RL}$ of the colloidal and solid forms of $\mathrm{CsPbBr}_{3}$ nanosheets. (a) Photographs of the concentrated colloid $(0.15 \mathrm{~g} / \mathrm{mL})$ under ambient light and an X-ray. The exposure time for the RL photo was set to 2 s. (b) Pulse height spectra of a standard commercial scintillator (Ce:LuAG) and a $\mathrm{CsPBBr}_{3}$ colloid. Note that the Compton edge for the colloidal scintillator is significantly weak. The RL spectra (c), peak (d), and intensity (e) were monitored during the transformation from a colloid to a solid in $12 \mathrm{~min}$. (f) Small-angle XRD shows the emergence of the superstructure during the drying process of the colloid.

In an attempt to quantify the RL light yield, a commercially available scintillator of cerium-doped lutetium-aluminum garnet (Ce:LuAG) single crystal was used as a reference standard, ${ }^{3}$ and exhibited a similar $\mathrm{RL}$ peak to colloidal $\mathrm{CsPbBr} 3$ nanosheets, both centered at $520 \mathrm{~nm}$ (Figure S7). Both samples were excited by $\gamma$-rays with an energy of $662 \mathrm{keV}$ ( ${ }^{137} \mathrm{Cs}$ source). The signal was fed into a multichannel analyzer (MCA) (ORTEC 926) and plotted into a pulse height spectrum (Figure 3b). Therefore, the channel number at the full energy peak represents the relative light yield of the scintillator. ${ }^{32}$ Importantly, the $\mathrm{CsPBBr}_{3}$ colloid exhibits a higher light yield $(\sim 21000$ 
photons/MeV) than the commercially available Ce:LuAG single-crystal scintillator ( $\sim 18000$ photons/MeV). In stark contrast to most of the conventional scintillators, the colloidal scintillator did not show any pronounced Compton edge, which may need further investigation.
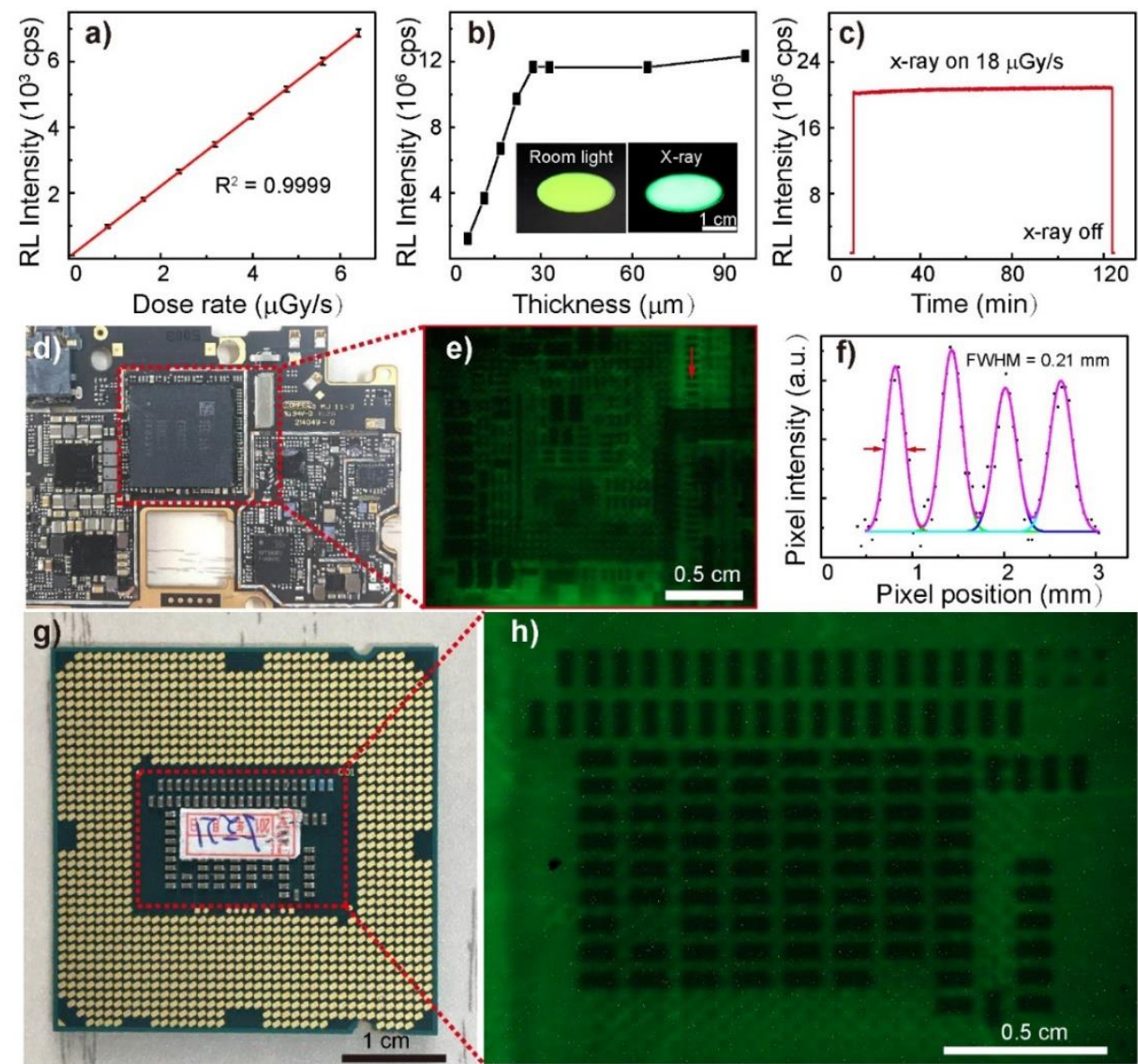

h)

Figure 4. Radiography property of the perovskite thin film comprising $\mathrm{CsPbBr}_{3}$ nanosheets. (a) The dose-dependence of the RL intensity shows a linear relationship. (b) The thickness dependence of the RL intensity suggests an optimal value at $25 \mu \mathrm{m}$, above which the added thickness is not able to gain more RL intensity. The insets show the photographs of a $25 \mu \mathrm{m}$-thick film under room-light and X-ray illumination, respectively. (c) Intensity record of the thin-film RL over 2-hour exposure of X-ray radiation at $18 \mu \mathrm{Gy} / \mathrm{s}$, showing a robust stability. (d) Photograph of a transistor panel in a cellphone. (e) The interior structure of the resin-covered panel (red dashed square) 
can be clearly revealed by the perovskite thin-film screen under our homemade radiography setup. (f) The point spread function (red line) of the intensity profile is fitted with a Gaussian function (blue line), and the full width at half-maximum (FWHM) is taken as the resolution. (g) Photograph of a standard CPU panel with a silicon chip integrated underneath. (h) X-ray imaging of the covered part (red dashed square) by the silicon chip, showing an unharmed resolution.

To monitor the RL change during self-assembly in real time, we used a fiber coupled spectrometer to record the $\mathrm{RL}$ of $\mathrm{CsPbBr}_{3}$ nanosheets during the transition from a colloid to a solid. As seen, the RL peak remains the same at $525 \mathrm{~nm}$ during the drying process (Figure 3d). Surprisingly, the RL of the colloidal scintillator is $20 \%$ higher than that of its solid form. The slight decrease in thin-film RL intensity is ascribable to the enhanced reabsorption between thin and thick nanosheets as the selfassembly proceeds. ${ }^{33}$ To elucidate the self-assembly process, a real-time XRD measurement was conducted (Figure $3 \mathrm{f}$ ). As the colloid solidifies, the peaks at $7.6^{\circ}$ and $11.4^{\circ}$ emerged and intensified as the time evolved, confirming the formation of a highly ordered superstructure (Figure S8). We also observed that the XRD peaks are significantly broadened due to the reduced grain size (Figure S2). Based on the Scherrer equation, the mean thickness of the nanosheets is calculated to be $2.95 \mathrm{~nm}$, which is in good agreement with the HRTEM result.

X-ray radiography is an imaging technique that uses the dose-dependence of scintillator RL to generate a phase contrast image. To assess the dose response of the perovskite film, we record the RL of a film (25 $\mu \mathrm{m}$ in thickness) under varied X-ray dose rates. The RL intensity shows an exceptional linearity to the dose rate in a broad 
range of 1 to $140 \mu \mathrm{Gy} / \mathrm{s}$ (Figure 4a and Figure S4). Importantly, the RL intensity increases with increasing thickness of the film in the range of 5-25 $\mu \mathrm{m}$ (Figure $4 \mathrm{~b}$ ). The intensity reaches a plateau when the thickness is above $25 \mu \mathrm{m}$, suggesting an optimal thickness of the active layer for the perovskite scintillator screen. This value is significantly smaller than those previously reported values, ${ }^{18}$ indicating the strong $\mathrm{X}$ ray stopping power of our thin film. Importantly, the perovskite film under investigation shows no radio-degradation effect under continuous X-ray irradiation for 2 hours (Figure 4c), exhibiting a long-term radio-stability. On a separate note, the lifetime of the perovskite scintillator is wavelength-dependent, ranging from 2 to $13 \mathrm{~ns}$ with varied wavelengths from 460 to $550 \mathrm{~nm}$ (Figure 2d). This is two orders of magnitude shorter than that of the conventional standard scintillator (NaI:Tl, decay time $\sim 200 \mathrm{~ns}$ ), which allows for significantly shortened deadtime for radiodetection devices. ${ }^{19}$ Holding these features, the perovskite thin film of stacked $\mathrm{CsPbBr}_{3}$ nanosheets qualifies for a potential candidate for radiography applications.

As a proof-of-concept experiment, we construct an X-ray imaging system with a projection configuration (Figure S5). By using a commercial camera and a 50-keV Xray source, the prototype X-ray imager can reveal the detailed structural information of a transistor panel under a resin cover (Figure 4e). To measure the resolution ability of our prototype, the point spread function of the intensity profile (red arrow in Figure 4e) is fitted with a Gaussian function. Impressively, such a simple prototype is able to provide a spatial resolution as high as $0.21 \mathrm{~mm}$ (Figure $4 \mathrm{f}$ ), mainly due to the crackfree virtue of the thin film. Apart from organic masks (i.e., resin), inorganic materials 
such as a silicon chip ( $\sim 300 \mu \mathrm{m}$ in thickness, Figure S6) can also be penetrated by Xrays without compromising any resolution ability (Figure $4, \mathrm{~g}$ and $\mathrm{h}$ ).

\section{CONCLUSIONS}

In conclusion, this work presented the room-temperature synthesis for a $\mathrm{CsPbBr}_{3}$ nanosheet colloid on the gram-scale. The concentrated colloid shows strong luminescence under both X-ray and UV light illumination. In addition, our colloidal perovskite nanosheets are readily cast into a crack-free thin film of a large area up to $72 \mathrm{~cm}^{2}$. The thin film consisting of self-assembled perovskite nanosheets not only exhibited a high PLQY but also showed long-term stability under continuous X-ray irradiation. The ensuing radiography prototype demonstrated a high-quality imaging ability with resolution down to $0.21 \mathrm{~mm}$. Our findings offer a colloidal scintillator to the screen industry, holding potential for low-dose radiography or even liquid scintillating displays.

\section{METHODS}

\section{Synthesis of $\mathrm{CsPbBr}_{3}$ Nanosheets}

$\mathrm{CsPbBr}_{3}$ nanosheets was synthesized by a significantly modified procedure reported by Konstantatos and co-workers. ${ }^{20}$ Typically, Cs precursor and $\mathrm{PbBr}_{2}$ precursor were prepared separately, and the reaction was initiated by injecting the latter into the former in a molar ratio of 4:1. Firstly, Cs precursor solution was prepared by dissolving $32 \mathrm{mg}$ CsAc in $1-\mathrm{mL} 1-\mathrm{PrOH}$ in a $20-\mathrm{mL}$ vial under stirring in air at room temperature, followed by addition of 6-mL Hex and 2-mL 1-PrOH. Secondly, $\mathrm{PbBr}_{2}$ precursor 
solution was prepared by dissolving $245-\mathrm{mg} \mathrm{PbBr}_{2}$ into a mixture solution of $0.45-\mathrm{mL}$ 1-PrOH, $0.45-\mathrm{mL}$ OcAc and $0.45-\mathrm{mL}$ OcAm at $90^{\circ} \mathrm{C}$ in air under vigorous stirring. Thirdly, the hot $\mathrm{PbBr}_{2}$ precursor was injected into Cs precursor swiftly under vigorous stirring at room temperature. The system turned green immediately, and the reaction completed in 2 minutes. The $\mathrm{CsPbBr} 3 \mathrm{NCs}$ were isolated by centrifugation at $4000 \mathrm{rpm}$, and the pellet was dispersed into 2-mL toluene. The synthesis has been successfully scaled up by 20 times, producing $\sim 3$ grams of $\mathrm{CsPbr}_{3}$ nanosheets in one single trial.

\section{RL and X-ray Imaging}

RL were obtained by an Edinburgh FS5 fluorescence spectrophotometer (Edinburgh Instruments Ltd., UK) equipped with a miniature X-ray source (AMPEK, Inc.). The Xray screen was made by drop casting colloid $(\sim 150 \mathrm{mg} / \mathrm{mL})$ onto a thin glass slide (150 $\mu \mathrm{m}$ in thickness). The thin-film screen was formed under ambient condition by slow evaporation of toluene. The photographs of X-ray-excited luminescence were acquired with a digital camera (Canon 600D with EF-S 35mm f/2.8 IS STM lens).

\section{ASSOCIATED CONTENT}

\section{Supporting Information}

The Supporting Information is available free of charge on the ACS Publications website at DOI: $\mathrm{xxx}$

Stability test, SEM cross-section image, Scherrer equation, dose-dependence of RL intensity, projection configuration of home-made prototype X-ray imager, RL spectra 
of $\mathrm{CsPBr}_{3}$ colloidal and $\mathrm{Ce}: \mathrm{LuAG}$ single crystal, large-area TEM image of nanosheet assemblies.

The authors declare no competing financial interests.

\section{AUTHOR INFORMATION}

\section{Corresponding Authors}

*Y. Z.: Email: ifc_zhangyh@ujn.edu.cn

*H. L.: Email: ifc_liuh@ujn.edu.cn

*O.F.M: Email: omar.abdelsaboor@kaust.edu.sa

\section{ACKNOWLEDGMENT}

This work was supported by both University of Jinan and King Abdullah University of Science and Technology (KAUST). Y. Z. has been supported by both National Natural Science Foundation of China (grant \# 21805111) and Taishan Scholar Fund. A. V. M. has been supported by the US NSF-CAREER grant \#1350800. He gratefully acknowledges travel support from CRDF Global at early stages of the work. 


\section{REFERENCES}

1. Lecoq, P., Development of New Scintillators for Medical Applications. Nucl. Instrum. .Methods Phys. Res. A 2016, 809, 130-139.

2. Weber, M. J., Inorganic Scintillators: Today and Tomorrow. J. Lumin. 2002, 100, $35-45$

3. Liu, S.; Feng, X.; Zhou, Z.; Nikl, M.; Shi, Y.; Pan, Y., Effect of $\mathrm{Mg}^{2+} \mathrm{Co}-$ Doping on the Scintillation Performance of Luag: Ce Ceramics. Phys. Status Solidi-R. 2014, 8, 105-109.

4. Nikl, M.; Yoshikawa, A., Recent R\&D Trends in Inorganic Single- Crystal Scintillator Materials for Radiation Detection. Adv. Opt. Mater. 2015, 3, 463-481.

5. Kanai, T.; Satoh, M.; Miura, I., Hot- Pressing Method to Consolidate $\operatorname{Gd}_{3}(\mathrm{Al}$, Ga) $)_{5} \mathrm{O}_{12}$ : Ce Garnet Scintillator Powder for Use in an X- Ray CT Detector. Intern. J. Appl.Cera.Technol. 2013, 10, E1-E10.

6. Pereira, M. d. C. C.; Madi Filho, T.; Berretta, J. R.; de Mesquita, C. H., Characteristics of the CsI: T1 Scintillator Crystal for X-Ray Imaging Applications. Mater. Sci. Appl. 2018, 9, 268.

7. Knoll, G. F., Radiation Detection and Measurement. John Wiley \& Sons: 2010.

8. Wurm, M.; Beacom, J. F.; Bezrukov, L. B.; Bick, D.; Blümer, J.; Choubey, S.; Ciemniak, C.; D’Angelo, D.; Dasgupta, B.; Derbin, A., The Next-Generation LiquidScintillator Neutrino Observatory Lena. Astropart. Phys. 2012, 35, 685-732.

9. Aberle, C.; Buck, C.; Gramlich, B.; Hartmann, F.; Lindner, M.; Schönert, S.; Schwan, U.; Wagner, S.; Watanabe, H., Large Scale Gd-Beta-Diketonate Based Organic Liquid Scintillator Production for Antineutrino Detection. J. Instrum. 2012, 7, P06008.

10. Lauck, R.; Brandis, M.; Bromberger, B.; Dangendorf, V.; Goldberg, M. B.; Mor, I.; Tittelmeier, K.; Vartsky, D., Low-Afterglow, High-Refractive-Index Liquid Scintillators for Fast-Neutron Spectrometry and Imaging Applications. IEEE Trans Nucl Sci. 2009, 56, 989-993. 
11. Kim, Y. C.; Kim, K. H.; Son, D.-Y.; Jeong, D.-N.; Seo, J.-Y.; Choi, Y. S.; Han, I. T.; Lee, S. Y.; Park, N.-G., Printable Organometallic Perovskite Enables Large-Area, Low-Dose X-Ray Imaging. Nature 2017, 550, 87.

12. Kovalenko, M. V.; Protesescu, L.; Bodnarchuk, M. I., Properties and Potential Optoelectronic Applications of Lead Halide Perovskite Nanocrystals. Science 2017, $358,745-750$.

13. Shamsi, J.; Rastogi, P.; Caligiuri, V.; Abdelhady, A. L.; Spirito, D.; Manna, L.; Krahne, R., Bright-Emitting Perovskite Films by Large-Scale Synthesis and Photoinduced Solid-State Transformation of $\mathrm{CsPbBr}_{3}$ Nanoplatelets. ACS Nano 2017, 11, 10206-10213.

14. Wei, H.; Fang, Y.; Mulligan, P.; Chuirazzi, W.; Fang, H.-H.; Wang, C.; Ecker, B. R.; Gao, Y.; Loi, M. A.; Cao, L.; Huang, J., Sensitive X-Ray Detectors Made of Methylammonium Lead Tribromide Perovskite Single Crystals. Nat. Photonics 2016, $10,333$.

15. Wei, W.; Zhang, Y.; Xu, Q.; Wei, H.; Fang, Y.; Wang, Q.; Deng, Y.; Li, T.; Gruverman, A.; Cao, L., Monolithic Integration of Hybrid Perovskite Single Crystals with Heterogenous Substrate for Highly Sensitive X-Ray Imaging. Nat. Photonics 2017, 11, 315 .

16. Yakunin, S.; Dirin, D. N.; Shynkarenko, Y.; Morad, V.; Cherniukh, I.; Nazarenko, O.; Kreil, D.; Nauser, T.; Kovalenko, M. V., Detection of Gamma Photons Using Solution-Grown Single Crystals of Hybrid Lead Halide Perovskites. Nat. Photonics 2016, 10, 585 .

17. Yakunin, S.; Sytnyk, M.; Kriegner, D.; Shrestha, S.; Richter, M.; Matt, G. J.; Azimi, H.; Brabec, C. J.; Stangl, J.; Kovalenko, M. V., Detection of X-Ray Photons by Solution-Processed Lead Halide Perovskites. Nat. Photonics 2015, 9, 444.

18. Chen, Q.; Wu, J.; Ou, X.; Huang, B.; Almutlaq, J.; Zhumekenov, A. A.; Guan, X.; Han, S.; Liang, L.; Yi, Z.; Li, J.; Xie, X.; Wang, Y.; Li, Y.; Fan, D.; Teh, D.; All, A.; Mohammed, O., F; Bakr, O.; Wu, T.; Bettinelli, M.; Yang, H.; Huang, W.; Liu, X., AllInorganic Perovskite Nanocrystal Scintillators. Nature 2018, 561, 88. 
19. Glodo, J.; Wang, Y.; Shawgo, R.; Brecher, C.; Hawrami, R. H.; Tower, J.; Shah, K. S., New Developments in Scintillators for Security Applications. Phys. Procedia 2017, 90, 285-290.

20. Di Stasio, F.; Christodoulou, S.; Huo, N.; Konstantatos, G., Near-Unity Photoluminescence Quantum Yield in $\mathrm{CsPbBr}_{3}$ Nanocrystal Solid-State Films via PostSynthesis Treatment with Lead Bromide. Chem. Mater. 2017, 29, 7663.

21. Brennan, M. C.; Herr, J. E.; Nguyen-Beck, T. S.; Zinna, J.; Draguta, S.; Rouvimov, S.; Parkhill, J.; Kuno, M., Origin of the Size-Dependent Stokes Shift in $\mathrm{CsPbBr}_{3}$ Perovskite Nanocrystals. J. Am. Chem. Soc. 2017, 139, 12201-12208.

22. Song, J.; Li, J.; Li, X.; Xu, L.; Dong, Y.; Zeng, H., Quantum Dot Light- Emitting Diodes Based on Inorganic Perovskite Cesium Lead Halides ( $\left.\mathrm{CsPbX}_{3}\right)$. Adv. Mater. 2015, 27, 7162-7167.

23. Yuko, T.; Keisuke, A.; Masahiro, R.; Kohei, S., Systematic Studies on Chain Lengths, Halide Species, and Well Thicknesses for Lead Halide Layered Perovskite Thin Films. Bull. Chem. Soc. Jpn. 2006, 79, 1607-1613.

24. Akkerman, Q. A.; Motti, S. G.; Kandada, A. R. S.; Mosconi, E.; D’Innocenzo, V.; Bertoni, G.; Marras, S.; Kamino, B. A.; Miranda, L.; De Angelis, F.; Petrozza, A.; Prato, M.; Manna, L., Solution Synthesis Approach to Colloidal Cesium Lead Halide Perovskite Nanoplatelets with Monolayer-Level Thickness Control. J. Am. Chem. Soc 2016, 138, 1010-1016.

25. Lindhoud, S.; Westphal, A. H.; van Mierlo, C. P.; Visser, A. J.; Borst, J. W., RiseTime of FRET-Acceptor Fluorescence Tracks Protein Folding. Int. J. Mol. Sci. 2014, $15,23836-23850$.

26. de Weerd, C.; Gomez, L.; Zhang, H.; Buma, W. J.; Nedelcu, G.; Kovalenko, M. V.; Gregorkiewicz, T., Energy Transfer between Inorganic Perovskite Nanocrystals. $J$. Phys. Chem. C 2016, 120, 13310-13315.

27. Wang, Y.; Zhu, Y.; Huang, J.; Cai, J.; Zhu, J.; Yang, X.; Shen, J.; Jiang, H.; Li, C., Cspbbr3 Perovskite Quantum Dots-Based Monolithic Electrospun Fiber Membrane as 
an Ultrastable and Ultrasensitive Fluorescent Sensor in Aqueous Medium. J. Phys. Chem. Lett. 2016, 7, 4253-4258.

28. Jagielski, J.; Kumar, S.; Wang, M.; Scullion, D.; Lawrence, R.; Li, Y.-T.; Yakunin, S.; Tian, T.; Kovalenko, M. V.; Chiu, Y.-C., Aggregation-Induced Emission in Lamellar Solids of Colloidal Perovskite Quantum Wells. Sci. Adv. 2017, 3, eaaq0208. 29. Rainò, G.; Becker, M. A.; Bodnarchuk, M. I.; Mahrt, R. F.; Kovalenko, M. V.; Stöferle, T., Superfluorescence from Lead Halide Perovskite Quantum Dot Superlattices. Nature 2018, 563, 671-675.

30. Kim, Y.; Yassitepe, E.; Voznyy, O.; Comin, R.; Walters, G.; Gong, X.; Kanjanaboos, P.; Nogueira, A. F.; Sargent, E. H., Efficient Luminescence from Perovskite Quantum Dot Solids. ACS Appl. Mater. Inter. 2015, 7, 25007-25013.

31. Zhang, Y.; Saidaminov, M. I.; Dursun, I.; Yang, H.; Murali, B.; Alarousu, E.; Yengel, E.; Alshankiti, B. A.; Bakr, O. M.; Mohammed, O. F., Zero-Dimensional $\mathrm{Cs}_{4} \mathrm{PbBr}_{6}$ Perovskite Nanocrystals. J. Phys. Chem. Lett. 2017, 8, 961-965.

32. Liu, C.; Hajagos, T. J.; Kishpaugh, D.; Jin, Y.; Hu, W.; Chen, Q.; Pei, Q., Facile Single- Precursor Synthesis and Surface Modification of Hafnium Oxide Nanoparticles for Nanocomposite $\gamma$ - Ray Scintillators. Adv. Func. Mater. 2015, 25, 4607-4616.

33. Liu, C.; Li, Z.; Hajagos, T. J.; Kishpaugh, D.; Chen, D. Y.; Pei, Q., Transparent Ultra-High-Loading Quantum Dot/Polymer Nanocomposite Monolith for Gamma Scintillation. ACS Nano 2017, 11, 6422. 